study in recent decades and, therefore, an investigation such as Mirabile's, that sheds new light on the "funzione Longhi," is altogether welcome. Mirabile's Scrivere la pittura is a significant contribution that provides us with a valuable vantage-point from which to understand Longhi's writing. The work further instils a new appreciation for his unique expressive modalities, his theory of verbal equivalences, and his key role within the history of twentieth-century Italian literature. It is therefore a pity that Mirabile's text is accompanied by only a few black and white illustrations, despite the numerous images treated in the book. A richer visual apparatus would have served better to represent the complex dialectic between word and painting in Longhi's world.

\title{
Giancarla Periti
}

University of Toronto

\section{Daniela Bartalesi-Graf. Voci dal Sud. New Haven: Yale University Press, 2011. Pp. 419. ISBN 9-780300-137446. n.p.}

Voci dal Sud è un testo per studenti di italiano a livello intermedio-avanzato. Ciò che lo rende particolarmente innovativo è il suo carattere interdisciplinare in cui lingua, storia, letteratura, arte, cinema e cultura forniscono allo studente un quadro completo dell'Italia, della sua lingua e della sua cultura.

Il conflitto tra Nord e Sud d'Italia, che affligge il paese dalla sua unificazione e che tutt'oggi è al centro di fervidi dibattiti, è affrontato principalmente tramite la lettura di alcuni brani del romanzo Cristo si è fermato a Eboli di Carlo Levi. Accanto al contributo letterario di questo scrittore, il testo ci presenta anche una serie di riproduzioni a colori di dodici dipinti di Carlo Levi, seguiti da una scheda critica sul film Cristo si è fermato a Eboli dell'acclamato regista Francesco Rosi, basato sul romanzo omonimo. Accanto a queste fonti primarie, Daniela BartalesiGraf ci fornisce anche una serie di risorse secondarie, completa di saggi critici sulla questione meridionale e su vari aspetti socio-economici e culturali del Sud d'Italia.

Voci dal Sud è un testo versatile che si addice, sia a corsi di letteratura, sia a corsi di cultura italiana: il volume offre all'insegnante la scelta di seguire un percorso letterario o un percorso culturale, o entrambi, senza trascurare l'apprendimento linguistico dell'italiano come lingua seconda. Il docente interessato al primo tipo di percorso si soffermerà sulla lettura dei brani di Cristo si è fermato a Eboli e degli altri brani letterari presenti nell'appendice. Il docente che invece vorrà seguire il secondo tipo di percorso, quello culturale, si soffermerà su quei capitoli e quei saggi che trattano la questione meridionale.

Il volume è diviso in sette capitoli dal tema e dalla struttura diversi, evitando così che lo studente possa trovarsi a svolgere sempre lo stesso tipo di attività, e permettendogli di variare continuamente il proprio metodo di apprendimento e il modo in cui viene valutato dall'insegnante. I capitoli si suddividono nel seguente modo:

Capitoli 1 e 5: contengono dei saggi storico-culturali sulla questione meridionale dall'unificazione ad oggi. Alla fine di ogni saggio vi sono delle domande per 
verificare la comprensione dello studente, e in fondo al capitolo vi è la sezione Ricerca, ragiona e discuti, in cui s'invita lo studente a fare delle ricerche in proprio e a discutere in classe alcuni aspetti degli argomenti in questione.

Capitolo 2: propone i brani di Cristo si è fermato a Eboli. La scelta di questo romanzo è sicuramente appropriata, non solo da un punto di vista contenutistico, ma anche da un punto di vista linguistico, in quanto il linguaggio usato da Carlo Levi risulta essere molto semplice e si adatta maggiormente alle competenze linguistiche di uno studente di italiano come lingua seconda.

Capitolo 3: in questo capitolo vengono presi in considerazione dodici dipinti di Carlo Levi che rappresentano le sue esperienze fatte durante l'esilio in Basilicata. Allo studente viene data l'opportunità di ammirare le riproduzioni di questi dipinti in un inserto a colori all'interno del capitolo. Le immagini riescono a dare all'alunno uno strumento in più per comprendere $\mathrm{a}$ fondo la sofferenza e l'umanità dei personaggi del romanzo leviano. Per aiutare nell'interpretazione dell'arte pittorica di Carlo Levi, viene fornita una guida alla lettura di un quadro nella sezione Punti di riflessione utili per leggere un quadro, seguita da una serie di domande specifiche su ogni dipinto, per una riflessione critica sull'opera. Anche in questo capitolo vi è spazio per ulterieri approfondimenti, nella sezione Soggetti per ricerche, approfondimenti o presentazioni orali.

Capitolo 4: dedicato interamente all'interpretazione del film di Francesco Rosi, Cristo si è fermato a Eboli. Il capitolo è completo di Stralci di interviste a Rosi e Le parole della critica, in cui, dopo aver letto delle brevi recensioni sul film, lo studente viene invitato a fare la propria recensione. Non manca poi un'attività di confronto tra romanzo e film.

Epilogo: questa sezione conduce ad una riflessione su tutto il materiale affrontato nel testo, mettendo in evidenza la sua rilevanza attuale e inquadrando i termini della questione meridionale nel Ventunesimo secolo.

Il testo può essere letto come una storia della questione meridionale dal suo inizio ad oggi, ma anche come una rivalutazione del contributo del Sud d'Italia allo sviluppo artistico e letterario dell'intera nazione.

Voci dal Sud è un volume completo che si adatta a varie metodologie d'insegnamento e di apprendimento (visuale, auditivo, ecc.). È un testo vivamente consigliato all'insegnante stanco dei tradizionali testi monodisciplinari e pronto ad affrontare nuovi temi sociali e culturali. Il volume infatti pone l'attenzione, non più sui maggiori centri culturali italiani come Roma, Firenze, Torino, eccetera, ma sulla realtà rurale di Aliano, un paesino della Basilicata, dove fu esiliato Carlo Levi; Aliano rappresenta la realtà rurale di tutta l'Italia di quel periodo e non solo della Basilicata.

Per finire Voci dal Sud offre all'insegnate un approccio interdisciplinare che permette all'allievo di sviluppare ed esercitare il suo pensiero analitico tracciando un parallelo tra film, letteratura e arte. 